\title{
Chapter 5 \\ Richardson and the Study of Dynamic Conflict Processes
}

\author{
Kelly M. Kadera, Mark Crescenzi and Dina A. Zinnes
}

\begin{abstract}
Lewis Fry Richardson made foundational contributions to the study of international relations. In this chapter, we examine his agenda-setting impact on the study of dynamics, time, and processes, especially conflict processes. We highlight the presence of Richardsonian dynamics in various formal and empirical models of peace and conflict. In so doing we emphasize the role of feedback and interactions in Richardson's models as well as other dynamic models, game-theoretic models, evolutionary game-theory and agent-based models, and quantitative empirical analyses. To show how these early foundations still inform research today, we then demonstrate how current research leverages dynamics to yield important insights concerning the origins and evolution of conflict such as when to expect norms of reciprocity to be present and to enable peace or exacerbate violence.
\end{abstract}

\subsection{Introduction}

Most important research questions in international politics concern processes with implicit time elements: how do crises evolve, when do hostile interactions become violent, how do shifts in trade affect alliances, when do regimes change? Answers inevitably require an explanation of how and why a process produces change over time. The pathbreaking work by Richardson (1960) helped us establish theories and methods for understanding time's role in IR. While Richardson was not the first to introduce the concept of time in international politics - see, e.g., the contemporaneous book by Sorokin (1957) on social and cultural change, which he linked to the emergence of war and the earlier work by Lanchester (1916) on attrition in combat ${ }^{1}-$ he provided a unique and valuable perspective by linking it to the broader notion of dynamics.

\footnotetext{
${ }^{1}$ For a comparison of the models of Lanchester and Richardson, see MacKay (2020, in this volume).
} 
Richardson's contributions are best seen through a comparison across various research traditions: game-theoretic models, evolutionary games and agent-based models (ABMs), differential equations (DEQ) models, and statistical analyses of time-structured data. We begin with a discussion of the concept of dynamics and then explore various approaches to time using illustrations from the literature. The differences across approaches highlight the significance of Richardson's contribution and suggest how scholars might incorporate it in future research.

\subsection{Richardsonian Dynamics: Time, Feedback, and Interactions}

The term dynamics is ubiquitous in the IR literature, and is often employed loosely to mean something along the lines of 'complicated and interesting.' Webster's New Collegiate Dictionary (1973) defines dynamics as 'the pattern of change or growth of an object or phenomenon'. Other definitions invoke terms like variation, forces, and continuous change. Time and process play essential roles. Change happens over time, according to some force acting on the phenomenon of interest. The word dynamic shares the same root as the Greek word dyne, which refers to 'the unit of force that would give a free mass of one gram an acceleration of one centimeter per second per second' (Webster's, 1973). As dyne is defined in terms of acceleration, its meaning is inherently tied to change over time.

Causal stories - arguments about what causes a particular variable to shift and take on different values - necessarily take place over time. Richardson's formulation of time, however, was more explicit. Although he sometimes used difference equations, in which a variable's value at time $t$ is a function of that variable (or others) at previous times (e.g., $t-1$ or $t-2$ ), his canonical work used DEQs, where forces (represented by first or higher order derivatives) cause variables to increase or decrease over time. ${ }^{2}$

Richardson's models not only portrayed an over-time dynamic process, they included feedback and were interactive. In his classic arms-race model, each nation's current level of arms has a negative impact (feedback) on its own subsequent rate of armament, decreasing the rate of change in armaments (see Smith, 2020, in this volume, for Richardson's full arms-race model). At the same time, the feedback mechanism is interactive. A rival nation's increasing level of armaments positively impacts one's own calculations, increasing a state's rate of change in armaments.

\footnotetext{
${ }^{2}$ Brown (2007) thoroughly discusses the differences and similarities of difference equation models and DEQ models, paying particular attention to the types of trajectories each produces.
} 


\subsection{Dynamics in Formal Theory Traditions}

Formal modeling traditions in IR - game theory, evolutionary games and ABMs, and DEQ models (which directly follow the Richardson tradition) - vary in how they feature dynamic elements. Here we sort out how each incorporates dynamics in its core characteristics and how those characteristics, or their absence, inform each tradition's understanding of global politics.

\subsubsection{Game Theory}

Game-theoretic models are commonly used in IR, and consist of players, actions (or strategies) they can choose, and payoffs associated with the outcomes produced by all possible combinations of actors' actions. Early game-theoretic models did not incorporate notions of time or process into any of these features of a game. In these simple, single shot, complete information games, players simultaneously choose their strategies and the outcomes, or Nash equilibria (NE), are immediately determined. Game-theoretic models have become considerably more complicated over time, though the basic structure remains.

Analyses of these models focus on finding equilibria, which are given by a list of the actions (strategies) each player would choose such that none would be better off by unilaterally changing to a different action (strategy). A sense of stickiness underlies the NE solution concept. Because no player has the incentive to unilaterally defect, a NE is inherently, or by its very design, stable. Osborne's description of a strategic form game highlights the absence of temporal features:

Time is absent from the model. The idea is that each player chooses her action once and for all, and the players choose their actions 'simultaneously' in the sense that no player is informed, when she chooses her action, of the action chosen by any other player. ... Nevertheless, an action may involve activities that extend over time, and may take into account an unlimited number of contingencies ... However, the fact that time is absent from the model means that when analyzing a situation as a strategic game, we abstract from the complications that may arise if a player is allowed to change her plan as events unfold: we assume that actions are chosen once and for all (Osborne, 2004: 14).

As such, scholars anticipate that players identify and immediately implement strategies congruent with the NE, and that such equilibria are stable. Without time, feedback is missing. However, interaction resides in the interdependent strategies that structure the game.

As game theory evolved, scholars incorporated temporal features. Most notably, modelers sequenced players' actions and introduced incomplete information. An actor could now observe what another chose in a previous move, enhancing the sense of feedback and interaction. Innovations such as the Subgame Perfect Equilibrium (SPE) concept eliminates equilibria where incredible threats are made in sequential play (e.g., Fight if the other player Backs Down). Stability still exists, 
as SPE are a subset of the NE and thus invoke the same stickiness inherent in the original approach.

Sequential play conveys a sense of progression over time. An SPE found through backwards induction can easily be represented by a highlighted path from first action to last (starting from the last move and inferred up the extensive form tree to the beginning move). Sequential games with perfect information yield SPE characterized by a complete list of plays for what each player will choose contingent on what state of the game they are at (e.g., what all the players have chosen up until that point in time). These choices are discrete in time and each player's strategy can be written down as an entire plan of play (e.g., Fight if the other Challenges, Accept if the other Offers to Negotiate). As such, an SPE is completely determined at the outset and we can find the SPE from a collapsed, simultaneous play version of the game in strategic form, though we will also get some equilibria that are incredible. Thus, time provides some new information and helps us eliminate some unmeaningful equilibria, but the model's strategic insights remain rather static.

Games with incomplete information provide more insight into uncertainties of strategic decision making, but their central solution concept, the Perfect Bayesian Equilibrium (PBE) retains the stickiness of the NE and SPE. The PBE 'stipulates that all strategies are sequentially rational and consistent with beliefs that are updated according to Bayes's rule wherever possible' (Thomas, Reed \& Wolford, 2016: 483). Players no longer know the payoffs of others, but instead assign probabilities to different payoff orderings. For example, Thomas et al. (2016) model rebel demands on governments, with war as one potential outcome. Rebels, $R$, do not know whether the government, $G$, can effectively fight or not. In turn, $R$ do not know G's preferences for fighting versus yielding. Finding the PBE involves identification of 'the range of player-types of $G$ that reject some proposal $x$ ' (Thomas et al., 2016: 506), $R$ 's choice of $x$ such that it 'never backs down' in the final stage of the game. Even though $R$ only knows $G$ 's type (or preferences), with some probability, and can update beliefs along the way, it decides at the outset what to do. Accordingly, 'In equilibrium, $R$ always chooses a demand for which it will refuse to back down after rejection, making credible its threat to fight by increasing the range of government types that reject' (Thomas et al., 2016: 484). From the start, each player chooses a strategy, or a complete way to play the game. Players instantly reach and stay at the PBE. Players' immediate movement to the equilibrium and its stickiness characterize prominent and common games and solution concepts in IR research. ${ }^{3}$

Bargaining models with iteration incorporate time more explicitly (e.g., Fearon, 1998). In such models, players would both rather reach an agreement than to continue fighting but prefer different agreements. Players incur costs from fighting, and the game's features allow us to ask whether and when players will strike a

\footnotetext{
${ }^{3}$ More advanced games incorporate features such as errors in strategy, and third players, and use more complex solution concepts.
} 
bargain, what it will look like, and what factors determine the answers to those questions. For example, Best \& Bapat's (2018) model reveals that insurgent leaders reject government offers when internal divisions threaten to undermine insurgent cohesiveness. In such models, time and timing matters more explicitly, but feedback is still elusive.

\subsubsection{Evolutionary Games and Agent-Based Models}

Evolutionary games explicitly incorporate time into game structures but relax classic game theory assumptions (such as rationality) and forfeit some strategic insights. Axelrod \& Hamilton (1981) develop PD tournaments in which actors adhere to one among several alternative strategies over the course of repeated plays. They notably demonstrate that cooperation can evolve because Tit-for-Tat (TFT), a 'nice' strategy that defects only if the opponent does so first, is evolutionarily stable. That is, when it dominates a population, TFT can survive invasion by mutant strategies. Analyses of evolutionary games focus on identifying evolutionarily stable strategies (ESS), but they differ from classic game theory strategies because they do not derive from actors' preferences; instead strategies are predetermined or inherited. When introducing replication dynamics, modelers also examine individuals' fitness for survival and temporal trends in the population of strategies.

Time plays at least two important roles in evolutionary games. First, results of past interactions feed back into the algorithm for an actor's current action. Second, patterns in the way (sub)populations of strategies rise and decline tell us whether and when we should empirically observe behavior like cooperation. Although few IR scholars use evolutionary games (but see, e.g., Johnson \& Toft (2013/14) on fluctuations in territorial conflict), many leverage the concepts in exploring cooperation mechanisms such as norm change (Finnemore \& Sikkink, 1998) or designing institutions for managing common pool resources (Ostrom, 1990).

Agent-based models similarly sacrifice traditional features of strategic interaction to capture how agent interactions produce temporal patterns at the aggregate level. ABMs add a social network to agent interactions: a grid system represents that network, agents are neighbors or distances separate them, and interactions depend on their closeness. Simulations reveal how populations of types of agents using various rules evolve, equilibria may not result, and may not be sticky. Scholars use ABMs to understand phenomena such as how coercion and emulation produce distinct temporal and geographic patterns in norm adoption (Ring, 2014) and the interplay between state formation and the severity of war (Cederman, 2003). ${ }^{4}$

\footnotetext{
${ }^{4}$ Cederman's (2003) agent-based model draws inspiration from Richardson's empirical work on power laws and wars. Cf. also Clauset (2020) and Spagat \& van Weezel (2020), both in this volume.
} 


\subsubsection{Differential Equations Models}

In contrast to classic game theory, but in concert with the spirit of evolutionary games and ABMs, Richardson and other scholars using DEQ models explicitly incorporate time, investigate more varied dynamic processes, and analyze their models with less focus on the equilibria themselves. Equilibria may or may not represent predictions of the model, but when they do, the issues concern stability. These distinctions translate into a greater focus on temporal features that characterize dynamic processes.

DEQ models specify at least one variable's values as a function of time. Typically, and in all the examples discussed in this section, a DEQ model uses more than one time-dependent variable, and the modeler builds a system of (first order) equations specifying how the change over time in one variable is driven by its own current value, other variables' current values, other variables' first (or higher) order derivatives, parameters, and the interactions of all of these elements. The resulting functions represent a variety of interdependencies and feedback loops.

A DEQ model's equilibrium is defined by the values of all the variables such that their first derivatives are zero. For example, in Richardson's arms race model, the equilibria are found by finding the value of $x$, nation X's level of armaments, and the value of $y$, nation Y's level of armaments, when $d x / d t=0$ and $d y / d t=0$. The no-change feature introduced by setting the first derivatives at zero seems, at first blush, to have some resemblance to a NE in game theory. However, not all equilibria are stable. That is, when a system in equilibrium is disturbed (e.g., shocked by an economic downturn that suddenly decreases one state's military spending), it might return to the equilibrium, and it might not. In the former case, we say the equilibrium is stable. ${ }^{5}$ In the latter, we say it is unstable. Stability is assessed by analyzing the behavior of the trajectories, or paths over time, near an equilibrium.

Trajectories are paths produced by interdependencies and feedback. Any system has an infinite number of trajectories, but they can be grouped into different types: some spiraling outward, indefinitely escalating upward (as in the arms race case of a Richardson model) or downward (as in the 'love race' case of the Richardson model); settling into stable equilibria (as in the case where the joint defense burden outweighs the joint threats in the Richardson model, so that the arms race ends); endlessly cycling but never reaching equilibria (as in population biology's predator-prey model or some of the models of demographic, fiscal, and elite models of state expansion and collapse in Turchin, 2003); approaching the equilibrium from some directions but move away from it in others, forming a saddle shape around the equilibrium (as in some versions of the Muncaster \& Zinnes, 1990, systemic hostility model); and so forth. Each type of trajectory is a little story about how the world might unfold, and savvy scholars can translate these types into substantively relevant vignettes about the causal process they are investigating. Multiple types of trajectories means multiple possibilities. In principle, once you

\footnotetext{
${ }^{5} \mathrm{An}$ analogy in empirical time-series research would be the notion of mean-reversion.
} 
know the initial conditions, the exact trajectory that the system takes is known, and assuming no exogenous shocks, the system is indeed deterministic.

Consider, for example, Kadera \& Morey (2008), which examines how the trade-offs of fighting and investing yield different outcomes under three types of competitions between states: peacetime rivalry, counter-industrial wars, and counter-force wars. They model the first derivative with respect to time of four variables: nation I's military spending $\left(m_{i}\right)$, nation I's level of resources $\left(r_{i}\right)$, nation J's military spending $\left(m_{j}\right)$, nation J's level of resources $\left(r_{j}\right)$. Each is a function of at least two of the others, and the exact functional form depends on the kind of competition I and $\mathrm{J}$ engage in. Their analysis of the model yields graphs of the four variables over time. The authors notice an interesting pattern over the course of counterforce war trajectories: I's and J's military expenditures flatten out and stay equidistant from each other, locking in a particular level for $m_{i}$ and for $m_{j}$, as well as for their difference, at all points in time. Kadera \& Morey label this type of trajectory a stalemate. They conclude that in counterforce wars, 'Both states are ... eventually able to replace the military power that is being destroyed from the fighting without significantly harming their economies. The traditional war trajectories demonstrate that each state is capable of continuing the conflict indefinitely and neither is able to gain an advantage over the other' (Kadera \& Morey, 2008: 167).

Differential equations models may not have equilibria, or current analytic methods may not allow the researcher to determine their explicit formulation. But the absence of equilibria does not negate the fact that the model can provide other, equally important insights such as trajectory behavior. Leveraging explicit equilibria values, Lee, Muncaster \& Zinnes (1994) identify types of triadic friend and enemy relationship structures that persist or evolve into different structures, and Toft \& Zhukov (2012) yield the conditions separating cases in which insurgency spreads across political subunits from those in which government coercion offsets transmission. Using simulations rather than explicit solutions or analytic identification of equilibria, Kadera, Crescenzi \& Shannon (2003) show how a strong global democratic community insulates nascent democracies from the autocratization effects of war \& Morey (2011) demonstrates how sudden upswings in fatalities shock a rivalry into termination by eroding support for continued hostilities.

Thus, two important points should be noted in comparing game theoretic and DEQ models. First, as Osborn (2004: 25) notes, 'Nash's theory concerns only equilibria; it has nothing to say about the path players' choices will take on the way to an equilibrium'. While game theoretic models reveal interesting interdependencies of decision making that are embedded in the final choices actors make, such as a rebel group making large demands that they know will be rejected by a government, in order to demonstrate their credibility (Thomas et al., 2016), DEQ models feature feedback that tells us about the type of route players or the system take and the speed of their travel. Second, the scholarly utility of a game theoretic model depends on whether it has few, identifiable equilibria; while the utility of a DEQ model relies less on the number or identifiability of equilibria and more on whether the trajectory behavior is substantively informative and interesting. In sum, 
if DEQ models represent what happens when actors do not 'stop to think', a famous characterization by Richardson, then game theoretic models represent what happens when actors do nothing but think.

\subsection{Dynamic Processes in Research Design and Empirical Analysis}

Although Richardson's work is most notable in its contribution to the modeling of international conflict, echoes of his approach can also be seen in quantitative empirical research. Action-reaction dynamics, for example, characterize early event coding and data. At the same time, scholars were grappling with the research design challenges of constructing statistical counterparts to Richardson arms race models (Schrodt, 1978). This early work on conflict processes continues to influence research today (e.g., Brandt et al., 2019) as is shown below in a brief overview.

\subsubsection{Early Work}

Early Richardsonian empirical work falls into two categories: (1) research focusing directly on Richardson's arms race model and (2) efforts to collect data on international events. Not long after Zinnes and others began working on theoretical models drawing from Richardson's work, empirical analyses of Richardson's work also began to emerge. Majeski \& Jones (1981), for example, revise and operationalize Richardson's equations in an attempt to better understand dyadic arms races, finding no evidence of an action-reaction process. Ward (1984) also leverages Richardson's equations when analyzing the arms race between the US and the USSR. Ward highlights the problem of simplistic operationalizations of the equations, particularly a confusion between arms stockpiles and current military budgets. He refocuses the work on perceived differences in stockpiles as a motivator for action and reaction, and in so doing finds that the US and the USSR participate 'in a reaction process in which each stimulates the other to spend more on their military establishments' (Ward, 1984: 202).

While the empirical work during this time is not explicitly driven by Richardson, it is possible to identify his influence more abstractly in the genesis of the events data sets begun in the 1970s. The World Events Interaction Survey (WEIS) codebook, for example, hints at dynamic processes when it refers to how the data 'reflect the flow of action and response between countries' (McClelland, 1978: 1), even though McClelland designed the WEIS project to merely catalogue the chronology of international events.

A few years later, the Conflict and Peace Data Bank (COPDAB) (Azar, 1980), was motivated largely by new perspectives on IR research, as laid out in Zinnes (1976). Whereas McClelland resisted scaling events, the COPDAB project ranked 
events along a single dimension of conflict and cooperation. These two event data sets (WEIS and COPDAB) stimulated a large body of scholarship. Although scholars identified serious challenges to statistically modeling and fitting Richardson arms race models (Schrodt, 1978), explorations of the broader notion of action-reaction dynamics developed by Zinnes (1980) and others (e.g., Gillespie et al., 1977) were well served by event data.

Goldstein (1992) used an expert survey to create a scale to migrate WEIS categories to a conflict-cooperation scale, launching a new wave of event-data research. This innovation enabled Goldstein \& Pevehouse (1997, see also Pevehouse \& Goldstein, 1999), for example, to examine dyadic and triadic reciprocity dynamics in Bosnia and Serbia, and Schrodt \& Gerner (1997) to examine phase shift dynamics in the Middle East. At the same time, Gerner \& Schrodt (1996) developed the first machine-coded event data generation processes, known as the Kansas Event Data System (KEDS), building on the infrastructure created by McClelland two decades prior.

\subsubsection{Current Empirical Research: An Illustration}

Quantitative empirical studies of conflict dynamics continue to develop. One contemporary empirical approach is seen in new research by Brandt, Freeman, Lin $\&$ Schrodt (2019). They apply time series models to analyze the conflict interaction patterns between dyads (pairs of states or actors) over time to pinpoint phases of reactivity versus phases of independent action. The focus is on three independent streams of conflict interactions between: Israel \& Palestinian groups, China \& Taiwan, and India \& Pakistan. Their search for phase shifts reveals that all three sets of interactions contain similar shifts between high and low-entropy, where entropy refers to the volatility and variance of actions and reactions within each dyad. ${ }^{6}$

Additionally, the authors find patterns that indicate the use of norms of reciprocity, wherein states react in kind to cooperative or conflictual events. Norms of reciprocation are useful in producing interactions conducive to peace and stability. The major substantive finding of the paper is that norms of reciprocity are more likely to govern behavior in low entropy phases. Thus, high-volatility phases create conditions under which leaders have a difficult time conveying and perceiving information about their intentions and the intentions of others. Reputations become harder to establish, signals are more difficult to send, and the problems relating to the analog of private information in bargaining environments become more pernicious.

Brandt et al. (2019) offer two important lessons: First, the explicit use of statistical methods to discern between different patterns of interactions over time could

\footnotetext{
${ }^{6}$ Based on our reading of Brandt et al. (2019), low-entropy is analogous to a stable equilibrium in a dynamic model, and high-entropy is analogous to an unstable equilibrium.
} 
uncover the complex dynamics of phase shifts and therefore help scholars understand when Richardson-like action-reaction processes occur. As such, the research design matters. Second, they find that the differences in the risk of severe conflict substantially differ across high and low entropy phases. Failing to take these differences into account runs the risk of pooling action-reaction patterns across high and low entropy phases and arriving at incorrect conclusions regarding the risk of war.

\subsection{Conclusions and Recommendations}

Richardson made a foundational contribution to the study of conflict - defining dynamics as an interactive, feedback-based, temporal process. Differential equations models explore the dynamics produced by interactive feedback mechanisms that highlight a rich variety of over-time patterns. In contrast, the focus of game theory is on interactions and expected interactions in the form of actor strategies and substantively important equilibrium solutions. Game-theoretic models do not explicitly capture interactions over time. Evolutionary games and ABMs marry some features of game theory with more dynamic elements that yield meaningful temporal patterns. In empirical work, theoretical dynamic processes are embedded in statistical research designs, while event data analyses utilize dynamic assumptions embedded in econometric models.

All the approaches reviewed here contribute to the study of world politics and contain an important lesson: explicitly incorporating dynamics and feedback mechanisms into theory, research design, and analysis provides valuable insight into causal processes (see Crescenzi \& Kadera, 2015). Our hope is that future scholars pay close attention to the role that dynamic processes can play in understanding international politics. Ignoring the impact of dynamics can mean the difference between finding or missing the answer to a research puzzle.

\section{References}

Axelrod, Robert \& William D Hamilton (1981) The evolution of cooperation. Science 211(4489): 1390-1396.

Azar, Edward E (1980) The conflict and peace data bank (COPDAB) project. Journal of Conflict Resolution 24(1): 143-152.

Best, Rebecca \& Nathan Bapat (2018) Bargaining with insurgencies in the shadow of infighting. Journal of Global Security Studies 3(1): 23-37.

Brandt, Patrick; John Freeman, Tse-min Lin \& Philip Schrodt (2019) A Bayesian time series approach to the comparison of conflict dynamics. Political Science Research and Methods, in press.

Brown, Courtney (2007) Differential Equations: A Modeling Approach. Los Angeles, CA: Sage.

Cederman, Lars-Erik (2003) Modeling the size of wars: From billiard balls to sandpiles. American Political Science Review 97(1): 135-150.

Clauset, Aaron (2020) On the frequency and severity of interstate wars. Ch. 10 in this volume. 
Crescenzi, Mark JC \& Kelly M Kadera (2015) Built to last: Understanding the link between democracy and conflict in the international system. International Studies Quarterly 60(3): 565-572.

Fearon, James (1998) Bargaining enforcement and international cooperation. International Organization 52(2): 269-305.

Finnemore, Martha \& Kathryn Sikkink (1998) International norm dynamics and political change. International Organization 52(4): 887-917.

Gerner, Deborah J \& Philip A Schrodt (1996) The Kansas event data system: A beginner's guide with an application to the study of media fatigue in the Palestinian intifada. Poster presented at the Annual Meeting of the American Political Science Association, San Francisco, CA.

Gillespie, John V; Dina A Zinnes, GS Tahim, Philip A Schrodt \& R Michael Rubison (1977) An optimal control model of arms races. American Political Science Review 71(1): 226-244.

Goldstein, Joshua S (1992) A conflict-cooperation scale for WEIS events data. Journal of Conflict Resolution 36(2): 369-385.

Goldstein, Joshua S \& Jon C Pevehouse (1997) Reciprocity, bullying, and international cooperation: Time-series analysis of the Bosnia conflict. American Political Science Review 91 (3): 515-529.

Johnson, Dominic DP \& Monica Duffy Toft (2013/14) Grounds for war: The evolution of territorial conflict. International Security 38(3): 7-38.

Kadera, Kelly M; Mark Crescenzi \& Megan Shannon (2003) Democratic survival, peace, and war in the international system. American Journal of Political Science 47(2): 234-247.

Kadera, Kelly M \& Daniel S Morey (2008) The trade-offs of fighting and investing: A model of the evolution of war and peace. Conflict Management and Peace Science 25(2): 152-170.

Lanchester, Frederick W (1916) Aircraft in Warfare: The Dawn of the Fourth Arm. London: Constable.

Lee, Sung-Chull; Robert Muncaster \& Dina Zinnes (1994) 'The friend of my enemy is my enemy': Modeling triadic internation relationships. Synthese 100(3): 333-358.

McClelland, Charles (1978) World Event/Interaction Survey: (WEIS), 1966-1978. Ann Arbor, MI: Inter-university Consortium for Political and Social Research.

MacKay, Niall (2020) When Lanchester met Richardson: The interaction of warfare with psychology. Ch. 9 in this volume.

Majeski, Stephen \& David Jones (1981) Arms race modeling: Causality analysis and model specification. Journal of Conflict Resolution 25(2): 259-288.

Morey, Daniel (2011) When war brings peace: A dynamic model of the rivalry process. American Journal of Political Science 55(2): 263-275.

Muncaster, Robert G \& Dina Zinnes (1990) Structure and hostility in international systems. Journal of Theoretical Politics 2(1): 31-58.

Osborne, Martin J (2004) An Introduction to Game Theory. New York: Oxford University Press.

Ostrom, Elinor (1990) Governing the Commons: The Evolution of Institutions for Collective Action. Cambridge: Cambridge University Press.

Pevehouse, Jon C \& Joshua S Goldstein (1999) Serbian compliance or defiance in Kosovo? Statistical analysis and real-time predictions. Journal of Conflict Resolution 43(4): 538-546.

Richardson, Lewis Fry (1960) Arms and Insecurity: A Mathematical Study of the Causes and Origins of War. Pittsburgh, PA: Boxwood.

Ring, Jonathan Jacob (2014) The Diffusion of Norms in the International System. PhD Dissertation. University of Iowa.

Schrodt, Philip A (1978) Statistical problems associated with the Richardson arms race model. Journal of Peace Science 3(2): 159-172.

Schrodt, Philip A \& Deborah J Gerner (1997) Empirical indicators of crisis phase in the Middle East, 1979-1995. Journal of Conflict Resolution 41(4): 529-552.

Smith, Ron P (2020) The influence of the Richardson arms race model. Ch. 3 in this volume.

Sorokin, Pitirim (1957) Social and Cultural Dynamics: A Study of Change in Major Systems of Art, Truth, Ethics, Law and Social Relationships. Reprinted, 1970. Boston, MA: Porter Sargent.

Spagat, Michael \& Stijn van Weezel (2020) The decline of war since 1950: New evidence. Ch. 11 in this volume. 
Thomas, Jakana L; William Reed \& Scott Wolford (2016) The rebels' credibility dilemma. International Organization 70(3): 477-511.

Toft, Monica Duffy \& Yuri M Zhukov (2012) Denial and punishment in the North Caucasus: Evaluating the effectiveness of coercive counter-insurgency. Journal of Peace Research 49(6): 785-800.

Turchin, Peter (2003) Historical Dynamics: Why States Rise and Fall. Princeton, NJ: Princeton University Press.

Ward, Michael D (1984) Modeling the USA-USSR arms race. Simulation 43(4): 196-203.

Webster's New Collegiate Dictionary (1973). Springfield, MA: Merriam.

Zinnes, Dina A (1976) Contemporary Research in International Relations: A Perspective and a Critical Appraisal. New York: Free Press.

Zinnes, Dina A (1980) Three puzzles in search of a researcher: Presidential address. International Studies Quarterly 24(3): 315-342.

Kelly M. Kadera, b. 1965, Ph.D. in Political Science (University of Illinois, 1993); Professor, University of Iowa (1993- ); Vice-President, International Studies Association (2016-17); current main interests: dynamics of conflict and democracy, conflict environments, gender and scholarship in International Relations, kelly-kadera@uiowa.edu

Mark J. C. Crescenzi, b. 1970, Ph.D. in Political Science (University of Illinois, 2000); Professor, University of North Carolina at Chapel Hill (1999- ); current main interests: reputation and learning in international politics, conflict environments and civil wars, dynamics of democracy, crescenzi@unc.edu.

Dina A. Zinnes, b. 1935, Ph.D. in Political Science (Stanford University, 1964); Professor of Political Science, Indiana University (1967-80), Merriam Professor of Political Science, University of Illinois (1980-2005), now emeritus. Founded and directed the Merriam Laboratory for Analytic Political Research (1986-2005). President of the International Studies Association (1980-81); Editor, American Political Science Review (1981-85), President, Peace Science Society (1989), zinnes@illinois.edu.

Open Access This chapter is licensed under the terms of the Creative Commons Attribution 4.0 International License (http://creativecommons.org/licenses/by/4.0/), which permits use, sharing, adaptation, distribution and reproduction in any medium or format, as long as you give appropriate credit to the original author(s) and the source, provide a link to the Creative Commons license and indicate if changes were made.

The images or other third party material in this chapter are included in the chapter's Creative Commons license, unless indicated otherwise in a credit line to the material. If material is not included in the chapter's Creative Commons license and your intended use is not permitted by statutory regulation or exceeds the permitted use, you will need to obtain permission directly from the copyright holder. 